\title{
CODEA: A FRAMEWORK FOR CO-DESIGNING ASSISTIVE TECHNOLOGIES WITH OCCUPATIONAL THERAPISTS, INDUSTRIAL DESIGNERS, AND END-USERS WITH MOBILITY IMPAIRMENTS
}

\author{
L. Aflatoony ${ }^{凶}$ and S. J. Lee \\ Georgia Institute of Technology, United States of America \\ $\bowtie$ leila.aflatoony@design.gatech.edu
}

\begin{abstract}
There are currently limited guidelines on how to design complex assistive technologies (ATs), which necessitates expertise beyond that possessed by designers, occupational therapists (OTs), or end-users. To address this issue, we conducted a series of four participatory workshops to study various configurations of OT-designer-user collaboration in co-designing do-it-yourself (DIY) ATs for an older adult with mobility impairment. We then proposed a specific co-design framework for such OT-designer-user collaboration.
\end{abstract}

Keywords: co-design, assistive technology, rapid prototyping, design process, collaborative design

\section{Introduction}

Occupational therapists (OTs) support individuals with physical impairments and disabilities (PwIDs) to maximize their independence in daily activities and participation in society through client-centered interventions. To achieve these goals, OTs focus on adapting the environment, Assistive Technologies (ATs), and/or tasks to fit the individual. While designing ATs is not necessarily the primary focus of the profession, many OTs engage in the adaptation of existing ATs as well as the creation of ATs through do-it-yourself (DIY) processes, especially when existing off-the-shelf AT solutions do not meet the clients' needs. Although digital fabrication and rapid prototyping tools potentially play an important role in designing custom ATs, they are currently underutilized by OTs, which is, in part, due to their unawareness as well as a perceived difficulty in creating 3D product geometries using CAD software.

In this paper, we propose a new framework, called "CoDEA", to guide the co-design process of occupational therapists, industrial designers, and end-users, in which we envision digital fabrication and rapid prototyping tools to play an integral role. The proposed framework was informed by a series of co-design workshops in which OTs, industrial designers, and end-users successfully collaborated to develop an assistive writing device for an end-user with post-polio syndrome. The complex task of designing ATs requires extensive knowledge, beyond the expertise of either discipline alone. While OTs' knowledge of the biological, biomechanical, and neurological underpinnings of function, as well as their understanding of the functional implications of disability, enables them to identify user needs, industrial designers' knowledge of ideation, prototyping, CAD modeling, 3D printing, and manufacturing can contribute to the tangible realization of AT design.

These workshops were motivated by the need to gain insight into the following questions: 1) how does an interdisciplinary team of occupational therapists, industrial designers, and end-user with diverse 
disciplinary expertise collaborate on designing assistive technologies? and 2) what are the opportunities and challenges in co-designing AT? Through observation and focus groups, we collected data on 1) the ways in which OT-designer-user's knowledge can be shared and communicated within the interdisciplinary team and 2) which means, media, and methods can be utilized to facilitate, transfer, and replicate skills and knowledge while co-designing ATs.

Our contribution speaks to the need for interdisciplinary collaborations on co-designing customdesigned ATs. Study findings revealed certain advantages of co-design workshops, participants' roles, and comparisons between occupational therapy and design practices. Furthermore, we determined a need for establishing a domain vocabulary and a shared repository of tools, methods, and materials for co-designing assistive technologies to support co-design practices. Finally, we propose a framework for maximizing OT-designer-user collaboration for co-designing custom ATs.

\section{Related work}

Although co-design with PwIDs remains relatively understudied, the importance of increasing the collaboration between designers and end-users (PwIDs) toward the creation of design solutions is well recognized. The co-design approach has been applied to address problems for which there are no feasible market solution due to their specificity (Thorsen et al., 2019; Buehler et al., 2014) or the fact that existing solutions are less than satisfactory due to a lack of fit with the specific needs, abilities, and contexts of users (Couvreur and Goossens, 2011; Thorsen et al., 2019). Furthermore, co-design has been described as a humanistic approach that extends beyond a mere functional need in order to develop holistic solutions that embody the emotional, social, and cultural aspects of users (SarmientoPelayo, 2015). In this endeavor, rather than being passive recipients of services, end-users are experts who actively shape the design process.

While some guidelines for engaging PwIDs in the co-design process have been reported, most work todate has focused on the two-way interaction between designers and PwIDs (Martinez et al., 2016; Sarmiento-Pelayo, 2015). There is currently less guidance for when a design problem requires expertise beyond those possessed by the designer, and necessitates collaboration with another profession. Although researchers have described some challenges with such interdisciplinary collaboration (ParryHill et al., 2017; Hofmann et al., 2016), only Couvreur and Goossens (2011) provided insight into how to structure the multi-entity process. In our study, we introduce an additional complexity to the co-design dynamic by bringing together two professions (OT and ID) and an end-user. By studying the process of this three-way (end user-OT-ID) collaboration, we aimed to shed light on how their different expertise is communicated, negotiated, and synthesized to create a design solution.

With the continued development of rapid prototyping technologies and the increasing interest of the healthcare field in exploring such advancements, the importance of co-design-enabled interdisciplinary collaboration will likely increase. Some early work explored the feasibility of clinicians learning 3D printing skills to produce simple assistive objects (Wagner et al., 2018; McDonald et al., 2016). While results were promising and suggested that $3 \mathrm{D}$ printing can be a viable tool for AT in real practice, a successful adoption of these $3 \mathrm{D}$ printing technologies on larger scales and for creating more complex, customized devices will necessitate partnering with designers to leverage their expertise. This collaboration with experts seems warranted given the documented difficulty of clinicians in gaining the advanced skills required for 3D printing (McDonald et al., 2016; Wagner et al., 2018).

\section{Study design and methodology}

\subsection{Co-design workshops: Prototyping writing ATs}

We held four participatory workshops, each of which lasted 1-3 hours, to study OT-designer-user collaboration in the development of assistive writing devices. Prior to this, we met with an occupational therapist (who did not participate in the workshop) to receive input and validate the workshop processes. A prototyping toolkit and materials to be used in the workshop were compiled after an online search to identify DIY materials commonly used by OTs in practice (e.g. Velcro, rubber bands, duct tape, sponge foam rubber, and tubing). Common industrial design prototyping 
materials were also provided (e.g. blue foam, thermoplastics, sanders, and cutting tools). To better understand the relative value of the different co-design approaches to AT solutions, each workshop followed a unique approach and consisted of different configurations of team members. The following table summarizes each workshop's approach, outcome, participants, team formation, and duration.

Table 1. Structures and configurations

\begin{tabular}{|c|c|c|c|c|}
\hline $\begin{array}{l}\text { Co-design } \\
\text { Workshops }\end{array}$ & $\begin{array}{l}\text { Workshop } 1 \\
\text { (Off-the-shelf } \\
\text { solutions) }\end{array}$ & $\begin{array}{c}\text { Workshop } 2 \\
\text { (Low-fidelity DIY } \\
\text { prototyping) }\end{array}$ & $\begin{array}{l}\text { Workshop } 3 \\
\text { (Designers as } \\
\text { technical } \\
\text { facilitators) }\end{array}$ & $\begin{array}{c}\text { Workshop } 4 \\
\text { (Designers as design } \\
\text { thinkers) }\end{array}$ \\
\hline Topic & $\begin{array}{l}\text { Intro to workshop: OT } \\
\text { approach to propose } \\
\text { AT solutions }\end{array}$ & $\begin{array}{l}\text { Do-it-yourself and } \\
\text { modification of } \\
\text { off-the-shelf } \\
\text { solutions }\end{array}$ & $\begin{array}{l}\text { Intro to digital } \\
\text { fabrication: } 3 \mathrm{D} \\
\text { printing method and } \\
\text { processes }\end{array}$ & $\begin{array}{l}\text { Intro to design stages } \\
\text { and process (design } \\
\text { thinking) }\end{array}$ \\
\hline Outcome & $\begin{array}{l}\text { Off-the-shelf AT } \\
\text { solutions }\end{array}$ & $\begin{array}{l}\text { Low-fi AT } \\
\text { prototypes }\end{array}$ & $\begin{array}{l}\text { 3D-printed AT } \\
\text { prototypes }\end{array}$ & $\begin{array}{l}\text { 3D-printed AT } \\
\text { prototypes }\end{array}$ \\
\hline Participants & $\begin{array}{l}4 \text { OTs } \\
1 \text { End-user }\end{array}$ & $\begin{array}{l}4 \text { OTs } \\
1 \text { End-user }\end{array}$ & $\begin{array}{l}4 \text { OTs; } 4 \text { Industrial } \\
\text { Designers; } 1 \text { End- } \\
\text { user }\end{array}$ & $\begin{array}{l}4 \text { OTs; } 4 \text { Industrial } \\
\text { Designers; } 1 \text { End- } \\
\text { user }\end{array}$ \\
\hline $\begin{array}{l}\text { Team } \\
\text { formation }\end{array}$ & $\begin{array}{l}\text { End-user working with } \\
4 \text { OTs at the same } \\
\text { time. }\end{array}$ & $\begin{array}{l}\text { End-user working } \\
\text { with } 4 \text { OTs } \\
\text { simultaneously. }\end{array}$ & $\begin{array}{l}\text { End-user working } \\
\text { with } 4 \text { teams (OT- } \\
\text { ID) simultaneously. }\end{array}$ & $\begin{array}{l}\text { End-user working } \\
\text { with } 1 \text { OT and } 1 \\
\text { Designer. }\end{array}$ \\
\hline Duration & 1 hour & 1 hour & 3 hours & 3 hours \\
\hline
\end{tabular}

The first two workshops involved OTs and the end-user. During the first workshop, following a comprehensive assessment of the user's abilities, limitations, and goals, OTs were tasked to identify available off-the-shelf AT solutions that would best meet the users' needs. In the second workshop, OTs created DIY-ATs, a common approach used by OTs for recreating or modifying existing assistive devices. Industrial designers joined the third and fourth workshops. In the third workshop, we envisioned their role to be that of a "technical facilitator," in which they assist and support the translation of OTs' and end users' ideas into tangible solutions via their sketching, prototyping, CAD modeling, and 3D printing skills. While these skills continued to be used in the fourth workshop, the industrial designers were prompted to be more intentional about following the design thinking process and actively contribute ideas to identify the user needs, brainstorm a wide variety of solutions, prototype iteratively, and develop a final prototype (Figure 1).
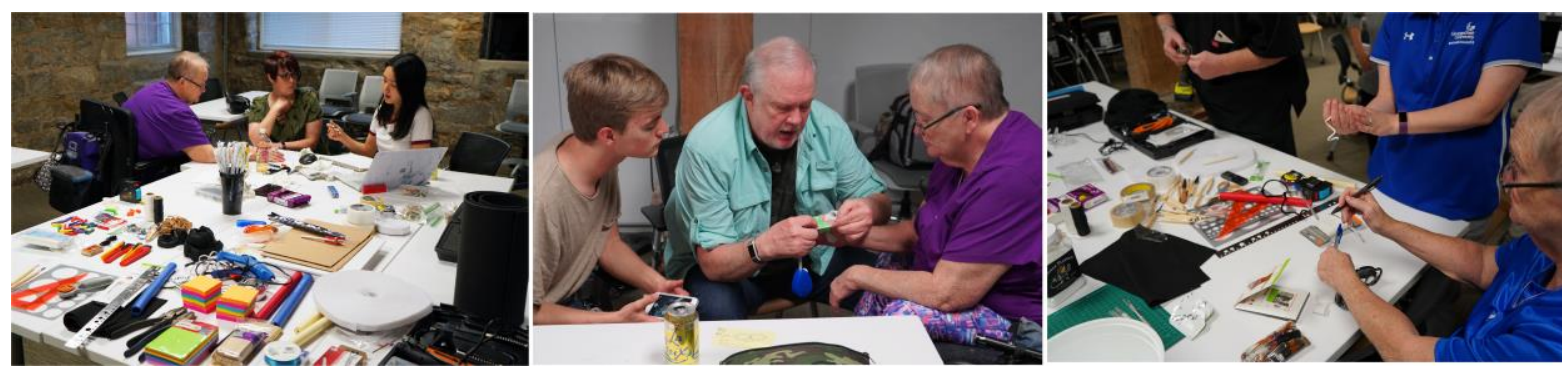

Figure 1. OT, designer, and user collaborate on developing assistive writing devices; From left to right: idea generation; clinical examination of user's hand; and prototyping ATs

Each of the four workshops began with a short presentation on design-related topics to familiarize the OTs with the designers' toolkit: prototyping materials, techniques, 3D digital fabrication, and design thinking. Multiple custom-designed AT prototypes were then developed and tested in order to provide continued insight into ideas, optimize user's assistive writing device, and leverage the benefits of 3D printing (rapid, iterative, and customizable). The main purpose of prototyping a custom AT was to reduce or eliminate pain for the user and enable her to continue participating in her educational activities. A thorough clinical 
examination by OTs revealed the design requirements for this writing AT: the device should neither touch her hand joints nor block them from bending, and it should support her free range of motion.

\subsection{Participant configuration}

Nine individuals, including four occupational therapists, four industrial designers (three senior undergraduates and one recent graduate), and one end-user with a mobility disability, participated in the study. Industrial design students were recruited through a department-wide recruitment e-mail. By attending a meeting of the state occupational therapy association and distributing a recruitment flyer to the authors' professional networks, we recruited the OTs. The end-user was a participant in a previous study and long-time supporter of aging and disability initiatives at Georgia Tech. The end-user was a female in her 70s who contracted polio as a child and utilized a wheelchair full-time. In addition to her post-polio syndrome, she experienced age-related mobility decline, which particularly affected her sensation and grasping strength. She experienced severe pain when engaging in fine motor activities that involved the extension of her hands (e.g. wrist extension during writing). For example, she had no issues in holding or grabbing a pen for a short time but could not maintain a firm grasp on a pen for an extended period of time without experiencing pain. As a result, she voiced a desire for a design solution that would enable her to hold and use a writing utensil for a longer amount of time without experiencing pain. This limitation affected other tasks as well: "I can't use a regular glass anymore; I have to use a glass with a handle". She actively attended continuing education classes and engaged in extensive notetaking, an effort fundamental to her success in studying but was, at the time, difficult to accomplish.

\subsection{Procedures for data collection}

We employed an observational approach to collect and analyse data regarding the participants' knowledge exchange, team dynamics, and tools and methods. Following a thorough observation process, we collected data from all workshop sessions, took notes, and organized the data into spreadsheets. We anonymized participants names for the designers (D1, D2, D3, D4), OTs (OT1, OT2, OT3, OT4), and end-user (U1) to protect their identities. In addition to structured observations, we conducted two focus groups (one for each group: OT and ID) and a semi-structured interview with the end-user at the conclusion of the workshop series to gain their opinions on the overall series quality, the strengths and weaknesses of each workshop, and the AT co-design process occurring via interdisciplinary teams. We conducted focus groups separately for each participant group to minimize any reluctance to share opinions in front of others. Each focus group session took between 20 minutes to one hour to complete. Each participant was compensated \$100-160 (varied due to different workshop attendance rates) for their participation in the workshops. Study procedures received ethics approval under an exempt review.

\section{Workshop results}

\subsection{Lists of OT-designer-user roles in co-designing ATs}

Our findings revealed that each participant group possessed a distinct sets of skills and expertise, informed by their training and prior experience, which influenced their approach as well as the overall dynamic of the team. For example, OTs' knowledge of human anatomy and biomechanics were instrumental in educating the designers and end-user on why writing was a difficult task for the enduser. At the very onset of the process, OTs assessed the source of this difficulty. Specifically, OT1 asked the end-user to simulate a writing task and asked questions to validate his assumptions on the exact location of her pain. The OTs were also interested in identifying other activities that caused similar pain, which they were able to infer from their experience working with clients with similar functional limitations. Through this inquiry process, the OTs determined that an important design goal for the tool in development would be to minimize thumb extension and flexion as well as the amount of grip required to operate the tool. While the focus of the workshops was on developing physical tools, the OTs also discussed other viable solutions, such as decreasing the extent of the end-user's writing through voice-to-speech detection technologies. 
The designers played a pivotal role in transforming these ideas into a tangible reality. While they were not always the ones suggesting ideas regarding the form factor and mechanisms of the tool, they were the experts in determining whether their ideas were practical enough for further pursuit. For example, OT1 suggested the idea of incorporating a retractable slide to allow for greater ease in donning and doffing the tool and asked D2 "is that possible?" D2 identified potential issues associated with the idea, including the complexity of the components that would be required to achieve the degree of movement desired; this eventually resulted in the team pursuing other solutions. The designers also supported the creation of solutions that were responsive to the OTs' need assessment, design requirements, and user feedback. Their role was particularly evident when multiple similar ideas were suggested simultaneously as well as when other participants presented incomplete ideas. For example, as the OTs recommended one AT design direction, they began to build on each other's ideas; thus, the designers helped provide a tangible structure to the conversation through sketching. As OT2 described:

"It was like I'm still talking but he [D1] is already drawing or doing something to kind of put those thoughts together. Whereas I would never try to draw anything like this. So, like we're on the same track but just how we operated was kind of interesting."

While expressing their lack of comfort with sketching and DIY prototyping themselves, many OTs expressed the benefit of having a visual representation of their ideas as they were being generated, as it allowed for a more effective, detailed discussion.

The end-user's involvement in the design process as an active collaborator (rather than a passive participant) improved the overall dynamics of the teams. For example, during the third workshop, the end-user shared her own design ideas with the teams. She also engaged in prototyping as a means to communicate her needs: "In this design, it's still holding your hand in a position where you are not going to be pinching but it's flexible so you can adjust it to whatever you need in order to use the pencil." She further stated: "I am thinking about the material, too. Because if the joint gets swollen (arthrosis) it would be more comfortable if we use a flexible fabric [...] what if we put this rubber around the utensil so it won't slide out?" In making decisions from a multitude of equally viable ideas, it was oftentimes the end-users' preferences or context-specific information, which only she could speak to, that determined the directions.

\subsection{Advantages of co-design for OTs, designers, and the end-user}

\subsubsection{OTs' reflection on co-design}

The OTs, designers, and end-user perceived the workshops as extremely beneficial. Specifically, OT1 described the workshops as a place in which "two different worlds [were] merging!" For the OTs, the co-design workshops presented them with the opportunity to be exposed to methods typically used by industrial designers. As one OT stated: “We have been exposed to design, we've talked about $3 D$ printers and even the material used to make it." Also, the co-design workshops enabled the OTs to observe the designers' processes, which helped encourage their future collaboration:
"One thing I came away with even after the very first workshop was that I was thinking about how nice and beneficial it would be for our patients or clients if we could work with designer other people like we work depending on the setting and we're able to work with other people in health care, but we tend to all exhibit the same thinking. But it would be so nice to actually have a designer available."

In addition to recognizing the benefit of working with design experts, the OTs regarded such collaboration as beneficial to their clinical practices, in which industrial designers could be partnered with clinics. OT4 asked: "How could there be a marriage between design consultants in health care? How can we get there?" Moreover, working in interdisciplinary teams enabled the OTs to be more creative during idea generation. OT4 described her experience as follows: "It's really nice to have that collaborative process, like a think tank, you know, because one person's idea kind of spark somebody else's thoughts or they're able to articulate it differently." OT3 stated that being exposed to other teams enabled her to think outside of the box: "I liked seeing everybody's thought process because 
they're so different from mine. I feel like I'm the least creative; but, seeing all the different OTs and all the different designers helped me see how everybody worked together to come up with ideas."

\subsubsection{Designers' reflection on co-design}

For the designers, the ability to leverage the OTs' clinical expertise was perceived as extremely beneficial, as it gave them more confidence in that fact that were beginning a design process with a set of robust, sophisticated design requirements. For example, during the fourth workshop, to educate D2, OT1 used an anatomical chart depicting the muscles and nerves of the hand: "This thumb here goes underneath the muscle and goes back up here, and that's what she's complaining hurts". The designers incorporated this knowledge from the OTs in their developing AT designs. As D2 stated:

"I learned a lot about the muscle anatomy of the hand, and I learned a fair bit about muscle dystrophy and post-polio, which is what [our end-] user was affected by... and the different ways in which the problem is worsened and solved in regard to that and what we can do to make that worse or make that not get worse."

D1 acknowledged the knowledge gained through the workshops: "Before this project, I was unaware of the challenges people with post-polio face on a day-to-day basis. Working with an end-user was a new experience for me and definitely got me outside my comfort zone." He further described his experience working with OT2: "Her knowledge of what positions and habits were detrimental to user were helpful in focusing on the best solution." D4 raised issues with the off-the-shelf ATs: "I learned they have a lot of problems that do not have a direct market solution. Also, I don't think they provide a solution for what to do when a user comes in with a unique problem. So that's a good opportunity to help them make new products."

On a slightly different note, D1 expressed a sense of fulfilment in working directly with the end-user: "While UI's condition limits her in many ways, she remained hopeful that we could come up with a way to preserve her ability to write. This motivated me throughout the process and makes me want to improve my design skills to better collaborate with users in the future." Also, D2 acknowledged the benefit of working with U1: "I thought it was very cool to actually find a person and say 'you're not just a design problem'. Like, you're a human and you have a story, and the story influences how we can solve your problem."

\subsubsection{End-user's reflection on the co-design}

Overall, the co-design workshops provided added value for the end-user beyond access to assistive writing tools. As U1 described, the workshops helped her became more aware of her own health conditions: "I enjoy working with people, but I also enjoy when I'm learning. I learned a lot and, you know, being a former peer supporter, I had a chance to actually learn more about the occupational therapy [and design] end of things. " She continued:

"Right now, I really need help because my arms are killing me and I now know what's going on with my nerves. I know that it's because of pinching so hard that it's made its way all the way up. So, I learned more about my own disability and what to look out for."

The end-user also spoke highly of the social interactions that occurred during the workshops, as well as the uniqueness of participating in something in which the whole goal was to make custom writing tools for her specifically. Throughout the workshop, we observed her in-depth participation and excitement in seeing the final ATs.

\subsection{The similarities and differences between designers' and OTs' practices}

The co-design workshops informed the OTs, designers, and the end-user about the tools, materials, and processes in occupational therapy and design disciplines. Such a process enabled them to learn from the other group's practices. While the industrial designers learned about OTs practices during the initial clinical assessment of the user's hand, Workshops 3 and 4 enabled the OTs to learn about the design process, identifying similarities between the two practices. As OT1 described, "I am going to 
say as much as we seem to be different these are really very similar approaches". He further described the occupational therapy process and then analysed and compared certain similarities between the two approaches (OT and design thinking) as follows:

- Client profile (equivalent of "empathize" in design thinking): OTs meet the client to learn about their needs (e.g. health status, experiences, and activities).

- Identify performance barriers (equivalent of "define" in design thinking): OTs identify client challenges and barriers (e.g. muscle fatigues, mobility issues).

- Explore the best solution direction (equivalent of "ideate" in design thinking): OTs explore different ideas to propose the best solution to the client.

- Introduce off-the-shelf ATs (equivalent of "prototype" in design thinking): OTs introduce available ATs in the market and adapt them according to the user's needs.

- Evidence-based practice (equivalent of "test" in design thinking): OTs document the effects of ATs based on the client's input.

Despite the above-mentioned similarities between the two practices, we observed unique contributions of each discipline to the co-design processes. For example, the OTs relied on the designers' expertise to convert their ideas into a tangible reality during the ideation and prototyping stages, whereas the designers had little or no input during the initial need identifying phase, trusting the OTs' expertise in understanding the users' needs. Another similarity was their perspectives on the importance of problem identification and how the "design-for-one" process allows for greater flexibility during this stage. In both disciplines, identifying "the right" problem is valued to such a degree that the extensive investment in resources will not occur until there is high confidence that a right problem has been identified. Given limited resources and time, problem identification should ideally be achieved early in the engagement with clients. Although fairly common in both disciplines, the phenomenon of clients changing their minds following the development of a solution poses several challenges. On the other hand, our workshops, which took a "design-for-one" approach and used rapid prototyping techniques, was better equipped for accommodating evolving needs. For example, as the OTs and designers were designing a writing AT for the user, the end-user requested the addition of a secondary function to support her other daily activities, such as crocheting and painting. While OT1 found clients' alteration of needs difficult, she recognized the importance of being able to meet those needs: "Especially in home health, you were there for one thing and then they throw out more things. You just can't be so rigid if you're a therapist."

\subsection{Domain vocabulary in co-designing ATs}

While both the OTs and industrial designers attempted to communicate their needs, opinions, and suggestions in the best ways possible, their choice of vocabulary was sometimes confusing for the other parties, especially in terms of domain-specific jargon or when the same terms was used by different disciplines with slightly different meanings. As D2 described: "You have to be on the same page, not only with each other if you're both working for somebody, but you have to speak and understand the same terms of the person you're helping."

Several strategies were used by all the participants to clarify and communicate their specific knowledge to the other parties. For example, the OT1 used a simplified language to describe the user's muscle function to D2: "Do you see this little cable here? That's her tendon." He further stated: "This thumb here goes underneath the muscle and goes back up here and that's what she's complaining hurts". Afterwards, D2 examined the user's hand muscles, and his understanding was solidified. Also, while the designers attempted to better understand the user's needs by asking the OTs questions, such as, "What is the healthiest position for her hand?", the term "healthy" was not specific enough for the OTs to understand the designer's question. Some designers described their experience with communicating their knowledge fairly positive (D1): "I gained a fair bit of experience on how to talk to people at a professional level in regard to design thinking and production things". Meanwhile, others found it challenging, such as D2: "I think explaining design terms that I've spent like a year learning, explaining that in an hour to someone and expecting them to have it and then come up with a product, was a little bit of a challenge." 


\subsection{Lack of common tools, methods, and materials for co-designing ATs}

The OTs and designers had a different preferences for the ways in which to communicate their ideas, which limited integration during the design process. As OT2 described: "It was like I'm still talking but he is already drawing or doing something to kind of put those thoughts together."

The OTs displayed greater comfort engaging with the end-user through long dialogues, preferring verbal conversations over physical creation, while the designers used sketching as a means to communicate their ideas (Figure 2). They also used familiar and tangible objects (e.g. pen, computer mouse) to quickly prototype and discuss ideas with the OTs. Despite encouragement, the OTs showed little interest in participating in the designers' ideation processes themselves, preferring to "leave it to the experts." Nonetheless, the OT participants expressed that, over the course of the workshop, they became increasingly confident using DIY materials to create low-fidelity AT solutions and share their ideas with the designers.

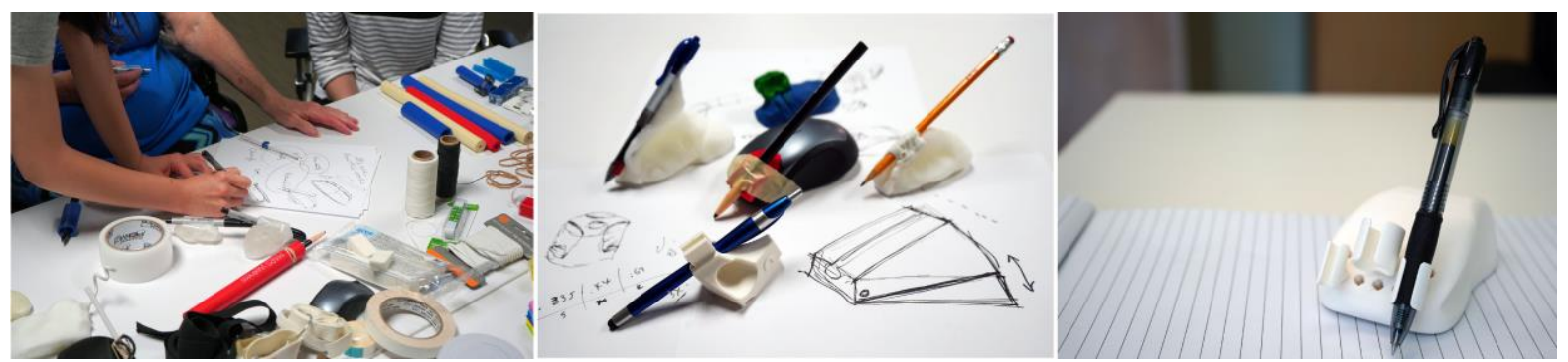

Figure 2. From left to right: An industrial designer communicates her ideas with an OT and the user through sketching; different materials and everyday objects were used to create lowfidelity prototypes of ATs; An assistive writing device inspired by computer mouse functions

The OTs and designers also differed in how they iterated through the prototyping and testing stages. The OTs' approach was characterized by a shorter iterative design cycle, that is, shorter spurts of prototyping testing and alternating between the two stages at a much higher rate than the designers. As they were working on making AT prototypes, the OTs were constantly testing the prototypes with the user to ensure a good fit. This process required the constant presence of the user. As OT2 described: "In designing ATs, it is important to always have the person present because their abnormalities in their hand position or joint position you can never recreate." OT4 confirmed OT2's statement and further reported: "You might need the client there throughout the process [...] like if it's just meant to be very custom, then you're going to need that person there almost the entire time." On the other hand, the designers preferred to spend more time generating and developing ideas before testing them with the user. While this resulted in a fewer number of encounters with the user, each encounter covered more ground. These differences meant that it was difficult for the end-user to anticipate when and by whom her input was required. Also, a lack of common knowledge about the tools and materials slowed down the design process, as described by D2: "Especially for materials. So, we'd be talking about plastic, and he would think I meant thermoplastics, but I was talking about the ABS plastic for print, and that took us a while to figure out."

\section{A framework for co-designing ATs with OT-designer-user}

The co-design workshops revealed how knowledge and skills from OTs, designers, and the end-user can be shared and how tools, methods, and materials can be used to support their collaboration. Our findings acknowledged the need for interdisciplinary collaboration in designing complex AT devices by revealing the advantages of co-design, roles and qualities of participation, and similarities and differences between OTs' and designers' practices. Unlike previous studies, which supported two-way interaction between designers and PwIDs (Martinez et al., 2016; Sarmiento-Pelayo, 2015), our approach facilitated OT-designer-user collaborations, and each group completed tasks within their respective various expertise. We did not compel members of one co-design group to perform tasks that the members of another group were experts in. This is in accordance with existing research on personal competencies. For instance, Drucker (2005) stated that an individual can be successful by 
improving her strengths but never by improving her weaknesses. Also, Inkson and King (2011) presented a "psychological contract" model on how individuals manage their careers, in which workers (or experts, such as designers and OTs) cultivate skills, expertise, and contacts they can often transfer easily from one organization to another. Accordingly, our co-designing framework allows for OTs and designers to stay within their respective areas of expertise, thus allowing them to nurture their respective expertise while not limiting participants' contribution to any limited role.

Furthermore, we observed the need for establishing a common vocabulary for OT-ID-user collaborations, which is in line with previous studies. According to March and Smith (1995), a domain's vocabulary "forms the special language and shared knowledge of a discipline". Kuhn (2012) also stressed its importance in describing problems and solutions within a domain. Besides, our findings highlighted common tools, methods, and materials used in OTs' and designers' disciplines and created a common toolkit repository to facilitate design discourse among the OTs, designers, and end-user. For example, while both OTs and designers were familiar with low-fidelity prototyping and DIY materials to create AT prototypes, they were also able to identify and use new, unfamiliar materials (e.g. thermoplastics).

Following the above-mentioned findings, we propose an initial framework for co-designing AT solutions with a OT-ID-user team (Table 2), which we call CoDEA. While this model bears some similarities with other design frameworks (e.g. design thinking, double-diamond model), it is unique in its foundation in observing the behaviour exhibited by the workshop participants in this study. In the CoDEA framework, while we encourage an ongoing collaboration of all team members throughout the co-design process, each party takes the lead during a different specific co-design stage in order to achieve the best AT solution. Due to the evolving nature of users' needs, the "problem identification" stage can evolve during the co-design process and continue into other stages.

Table 2. The CoDEA framework for co-designing DIY-ATs with three groups: occupational therapists (OTs), industrial designers (IDs), and end-users

\begin{tabular}{|lll|l|l|l|}
$\begin{array}{l}\text { Co-design } \\
\text { Stages }\end{array}$ & $\begin{array}{l}\text { 1. Clinical Need } \\
\text { Dentification }\end{array}$ & $\begin{array}{l}\text { 2. AT Problem } \\
\text { Identification }\end{array}$ & $\begin{array}{l}\text { 3. Explore AT } \\
\text { Solutions }\end{array}$ & $\begin{array}{l}\text { 4. Printed AT } \\
\text { Solutions }\end{array}$ & $\begin{array}{l}\text { 5. Examine } \\
\text { AT } \\
\text { Solutions }\end{array}$ \\
\hline Participants & ID-user & ID-user & OT-user & OT-user & ID-user \\
\hline Leader & OT & OT & ID & ID & OT \\
\hline $\begin{array}{l}\text { Co-design } \\
\text { processes }\end{array}$ & $\begin{array}{l}\text { OTs work with end- } \\
\text { users to examine } \\
\text { their health status, } \\
\text { experiences, and } \\
\text { activities. }\end{array}$ & $\begin{array}{l}\text { OTs identify } \\
\text { users' } \\
\text { performance and } \\
\text { activity barriers. }\end{array}$ & $\begin{array}{l}\text { Designers } \\
\text { explore } \\
\text { different AT } \\
\text { designs based } \\
\text { on OTs and } \\
\text { end-user } \\
\text { feedback. }\end{array}$ & $\begin{array}{l}\text { Designers } \\
\text { develop CAD } \\
\text { files, 3D } \\
\text { models, and }\end{array}$ & $\begin{array}{l}\text { OTs } \\
\text { 3D-printed AT } \\
\text { solutions. AT } \\
\text { solutions by } \\
\text { observing } \\
\text { users }\end{array}$ \\
$\begin{array}{llll}\text { interacting } \\
\text { with them. }\end{array}$ \\
\hline
\end{tabular}

\subsection{Comparison with other models}

As OTs, designers, and the user developed prototypes, they came to further understand certain design problems and, thus, requirements. In particular, the end-user presented new ideas for "improving" the AT design features in every workshop. Similar hypotheses has been brought up by Dorst and Cross (2001) and confirms that design problems and their solutions can "co-evolve" from experienced designers. Also, Cross (2001) argued that "designers move rapidly to early solution conjectures and use these conjectures as a way of exploring and defining problems and solutions together." In addition, we argue that co-designing ATs is an iterative process, with a shorter prototyping-testing design cycle, which enables the simultaneous assessment of AT solutions and end-users. This is in-line with the "develop phase" of the Double Diamond model (UK Design Council, 2019), a phase of divergent thinking that concentrates on generating multiple concepts through iteration.

\subsection{Limitations and future work}

Our proposed framework, while informed by our work, which directly engaged the relevant stakeholders in co-design processes, has several limitations. Although we were cognizant of the somewhat 
serendipitous nature of brainstorming, for time-keeping purposes, it was necessary to establish clear expectations to keep the discussions on topic. This requirement may have posed restrictions on behaviours that may have actually resulted in stronger dynamics in the long term. In addition, we observed a relatively limited divergence in ideas throughout the workshop, suggesting that more investigation is needed to understand the ways in which to support the interdisciplinary dynamic and encourage creative exploration. In addition, the pairing of OTs with extensive clinical experience and industrial designers currently undergoing or having recently completed training may have introduced factors that are not generalizable across all the teams. Future work should include validating and refining this framework by involving additional end-users as well as investigating strategies to overcome current barriers that limit effective collaboration.

\section{References}

Buehler, E., Hurst, A. and Hofmann, M. (2014), "Coming to grips: 3D printing for accessibility", Proceedings of the 16th international ACM SIGACCESS conference on Computers \& accessibility (ASSETS '14), ACM, New York, NY, USA, pp. 291-292. https://doi.org/10.1145/2661334.2661345

Couvreur, L.D. and Goossens, R. (2011), "Design for (every)one: co-creation as a bridge between universal design and rehabilitation engineering", CoDesign, Vol. 7 No. 2, pp. 107-121. https://doi.org/1 $0.1080 / 15710882.2011 .609890$

Cross, N. (2001), "Design cognition: results from protocol and other empirical studies of design activity", In: Eastman, C., Newstatter, W. and McCracken, M. (Eds.), Design knowing and learning: cognition in design education, Elsevier, Oxford, UK, pp. 79-103. https://doi.org/10.1016/B978-008043868-9/50005-X

Dorst, K. and Cross, N. (2001), "Creativity in the design process: co-evolution of problem-solution", Design studies, Vol. 22 No. 5, pp. 425-437. https://doi.org/10.1016/S0142-694X(01)00009-6

Drucker, P.F. (2008), Managing oneself, Harvard Business Review Press.

Hofmann, M. et al. (2016), "Clinical and Maker Perspectives on the Design of Assistive Technology with Rapid Prototyping Technologies", Proceedings of the 18th International ACM SIGACCESS Conference on Computers and Accessibility (ASSETS '16), ACM, New York, USA, pp. 251-256. https://doi.org/1 $0.1145 / 2982142.2982181$

Inkson, K. and King, Z. (2011), "Contested terrain in careers: A psychological contract model", Human relations, Vol. 64 No. 1, pp. 37-57.

Kuhn, T.S. (2012), The structure of scientific revolutions, University of Chicago press.

March, S.T. and Smith, G. (1995), "Design and Natural Science Research on Information Technology", Decision Support Systems, Vol. 15 No. 4, pp. 251-266. https://doi.org/10.1016/0167-9236(94)00041-2

McDonald, S. et al. (2016), "Uncovering Challenges and Opportunities for 3D Printing Assistive Technology with Physical Therapists", Proceedings of the 18th International ACM SIGACCESS Conference on Computers and Accessibility (ASSETS '16), Association for Computing Machinery, New York, NY, USA, pp. 131-139. https://doi.org/10.1145/2982142.2982162

Martinez, S. et al. (2016), "Building Bridges Between User and Designer: Co-creation, Immersion and Perspective Taking", In: Bucchianico G., D. and Kercher, P. (Eds.), Advances in Design for Inclusion. Advances in Intelligent Systems and Computing, vol 500, Springer, Cham.

Parry-Hill, J. et al. (2017), "Understanding Volunteer AT Fabricators: Opportunities and Challenges in DIY-AT for Others in e-NABLE", Proceedings of the 2017 CHI Conference on Human Factors in Computing Systems (CHI '17), ACM, New York, NY, USA, pp. 6184-6194. https://doi.org/10.1145/3025453.3026045

Sarmiento-Pelayo, M.P. (2015), "Co-design: A central approach to the inclusion of people with disabilities", Revista de la Facultad de Medicina, Vol. 63 No. 1, pp. 149-154. https://dx.doi.org/10.15446/revfac med.v63n3sup. 49345

Thorsen, R., Bortot, F. and Caracciolo, A. (Jul 16 2019), "From patient to maker - a case study of co-designing an assistive device using 3D printing", Assistive Technology, pp. 1-7. https://doi.org/10.1080/104 00435.2019.1634660

UK Design Council. (2019), What is the framework for innovation? Design Council's evolved Double Diamond. Retrieved from https://www.designcouncil.org.uk/news-opinion/what-framework-innovation-designcouncils-evolved-double-diamond

Wagner, J.B. et al. (2018), "Three professions come together for an interdisciplinary approach to 3D printing: occupational therapy, biomedical engineering, and medical librarianship", Journal of the Medical Library Association: JMLA, Vol. 106 No. 3, pp. 370-376. https://doi.org/10.1007/978-3-319-41962-6_11 